\title{
A New Energy Efficient Target Detection Scheme for Pervasive Computing*
}

\author{
Thanh Hai Trinh and Hee Yong Youn ${ }^{* *}$ \\ School of Information and Communication Engineering, \\ Sungkyunkwan University, 440-746, Suwon, Korea \\ hai9381@skku.edu, youn@ece.skku.ac.kr
}

\begin{abstract}
Energy is one of the critical constraints for the applications of sensor network. In the earlier target detection and tracking algorithm power saving is achieved by letting most of the non-border sensor nodes in the cluster stay in hibernation state. However, the border sensor nodes consume a significant amount of energy since they are supposed to be on all the time for target detection. In this paper we propose a new target detection scheme which lets the border sensor nodes be on shortly one after another in a circular fashion to minimize the energy consumption. Computer simulation shows that the proposed scheme can significantly reduce the energy consumption in target detection and tracking compared to the earlier scheme.
\end{abstract}

Keywords: Cluster, energy saving, target detection, pervasive computing, wireless sensor network.

\section{Introduction}

With advances in computation, communication, and sensing capabilities, large scale sensor-based distributed environments are emerging as a predominant pervasive computing infrastructure. One of the most important areas where the advantages of sensor networks can be exploited is tracking mobile targets. The scenarios where the network may be deployed can be both military (tracking enemy vehicles and detecting illegal border crossings) and civilian [1]. In developing the sensor networks for target tracking, battery power conservation is a critical issue.

In the sensor network a large number of sensor nodes carry out a given task. In [2], a distributed, randomized clustering algorithm was given to organize the sensors in the wireless sensor network into a hierarchy of clusters with an object of minimizing the energy spent for the communication with the information center. The problem of tracking the targets using the sensor network has received attention from various angles. In [5], the authors consider the case where a set of $k$ targets need to be tracked with three sensors per target from the viewpoint of resource requirement. They identified that the probability that all targets can be assigned three unique sensors shows

\footnotetext{
* This research was supported in part by the Ubiquitous Autonomic Computing and Network Project, $21^{\text {st }}$ Century Frontier R\&D Program in Korea and the Brain Korea 21 Project in 2006.

** Corresponding author.
} 
phase transition properties as the level of communication between the sensors increases. In [7], the information driven sensor collaboration mechanism is proposed. In this mechanism the measures of information utility are used to decide future sensing actions. Collaborative signal processing aspects for target classification in sensor networks is addressed in [8]. The techniques for locating targets using a variety of mechanisms have been proposed in [9-11]. In [3], the authors present a prediction based approach, called localized predictive, for power efficient target tracking. In [4], tracking based on a distributed and scalable predictive algorithm called the distributed predictive tracking algorithm (DPT) is proposed. The DPT algorithm divides the sensor nodes in a cluster into border sensor nodes and non-border sensor nodes, and achieves power saving by letting most of the non-border sensor nodes stay in hibernation state. The border sensor nodes are awake all the time to detect the targets.

In this paper we propose a scheme detecting the targets in the sensor network, which can save the energy of border sensor nodes as well. One after another in the circular fashion, the border sensor nodes are activated to detect the target moving into the sensing area and then returns to hibernation mode. Consequently, the energy spent for detecting the target with the proposed scheme is much smaller than that of the DPT algorithm. If there exist several targets simultaneously moving into the sensing area, several messages will be sent to the cluster head. Therefore, we consider the relation between the processing time in the cluster head and velocity of the target movement to model the target detectability and tracking capability of a sensor network. Computer simulation shows that the proposed scheme can significantly reduce the energy consumption compared to the DPT algorithm for the targets of typical speeds.

The rest of the paper is organized as follows. In the following section we present a review of the DPT algorithm to identify the power problem. Section 3 presents the proposed detection scheme, and Section 4 discusses the simulation results. Finally, Section 5 presents the concluding remarks and future work.

\section{The Related Work}

The distributed predictive tracking (DPT) [4] algorithm employs the distributed and scalable prediction based approach to accurately track mobile targets using a sensor network. The fundamental guideline followed throughout the design of the DPT algorithm was to keep it as simple as possible. The DPT algorithm uses a cluster-based architecture for scalability and robustness. Given a target to track, the protocol provides a distributed mechanism for locally determining an optimal set of sensors for tracking. Only the nodes are then activated to minimize the energy consumption. Most of the sensors stay in hibernation mode until they receive an activation message from their cluster head. This is made possible by predicting the target's next location.

The DPT algorithm distinguishes the border sensor nodes from the non-border sensor nodes in terms of their operation. While the border sensors are required to be awake all the time in order to detect any target entering the sensing region, the nonborder sensors hibernate unless they are asked to wake up by their cluster head. Hence, the energy of border sensor nodes will decrease quickly, while the task of border sensor nodes is critical for target detection. Therefore, an approach saving the energy of border sensor nodes as much as possible without reducing the target detectability is needed. 


\section{The Proposed Scheme}

In this section we present the proposed scheme allowing energy efficient target detection and tracking with a sensor network. The scheme allows the border sensor nodes to minimize the energy consumption on target detection.

\subsection{Assumptions}

We first discuss the assumptions made by the proposed scheme. It assumes a clusterbased architecture for the sensor network [2,6]. Initially, all the border sensor nodes have information on their neighboring sensor nodes for their identity, location, and energy level. Each cluster head also has such information on the sensor nodes belonging to its cluster. When tracking a moving target, it decides which border sensor nodes detect the presence of a target. The assumptions on the sensor nodes are given below.

- $\quad$ Each border sensor is activated for $\Delta T$ time, and then returns to hibernation mode.

- The border sensor nodes are assumed to be uniformly distributed over the sensing region and the number of sensor nodes is large.

- Let $d$ be the distance between two neighboring border sensors. $d$ is smaller or equal to the radius of the sensible area of a sensor, $r$. If $d$ is larger than $r$, the intersecting area between two neighboring sensors will become small and the detection probability decreases accordingly. (Refer to Figure 1.)

- The targets originate outside the sensing area and then move into the area with constant velocity.

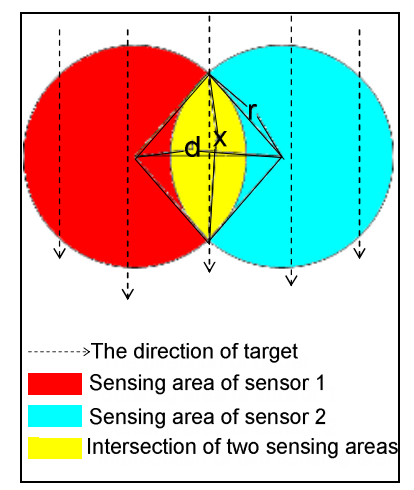

Fig. 1. Intersection of two sensing areas

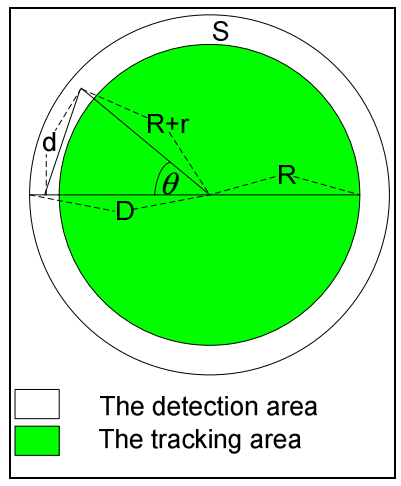

Fig. 2. The structure of a cluster

\subsection{The Detection Algorithm}

When targets move into the sensing area, the border sensors detect the presence of them and then inform the cluster head on it. The proposed mechanism is explained using the parameters listed in Table 1. 
Table 1. The parameters used in the proposed mechanism (Refer to Figure 2)

\begin{tabular}{|l|l|}
\hline Parameter & \\
\hline$V$ & The velocity of target \\
\hline$T$ & The period a border sensor is turned on and off \\
\hline$N$ & The number of border nodes \\
\hline$r$ & The radius of the sensing area of a sensor node \\
\hline$d$ & The distance between two neighboring border sensor nodes \\
\hline$R$ & The distance from the cluster head to the edge of detection area \\
\hline$D$ & The radius of the cluster, which is $R+2 r$ \\
\hline$\theta$ & The angle at the cluster head made by two neighboring border sensors \\
\hline$S$ & The detection area covered by the border nodes \\
\hline$\lambda$ & The sensor density \\
\hline
\end{tabular}

The proposed scheme divides the detection period, $T$, into equal interval of $\Delta T$ given by: $\Delta T=T / N$. In other words, every border node turns on for $\Delta T$ time in every $T$ time unit. In the DPT algorithm, all the border sensor nodes are activated to detect the targets. If there is only one target moving into the sensing area, a significant amount of energy will thus be wasted. Therefore, we propose to let only one border sensor is activated during $\Delta T$ and others are put in hibernation mode. Once a border sensor wakes up during $\Delta T$, it then stays in hibernation mode during $(N-1) \Delta T$. This process repeats continuously with a period of $T$. By letting the border sensor nodes on and off fast enough, any target penetrating the border can be detected regardless of the entering direction. However, if a target moves faster than a certain threshold, it cannot be detected since the border sensor node in charge of it might still be in hibernation mode. Therefore, we need to decide the time $T$ of one round of detection period according to the maximum velocity of target movement, $V_{\max }$.

Assume that the border sensors are deployed with Poisson distribution with a parameter $\lambda S$. In Figure 2, since the number of border sensors is very large, $\theta$ is very small and

$$
\sin \theta \approx \theta=\frac{2 \pi}{N}=\frac{d}{r+R}
$$

For conservative modeling, assume that the target moves into the sensing area in the right angle and proceeds in the straight line (thus shortest path). Let us denote $x_{\min }$ the length of the path the target takes. Since the target has a constant velocity, the period, $T$, can be calculated in the following equation.

$$
T=\frac{x_{\text {min }}}{V_{\text {max }}}
$$

As shown in Figure 1, the minimum length $x_{\min }$ results in when the target moves through the intersection area of two neighboring border sensors. If the intersection area is small, the target detection probability will be low accordingly. To guarantee a reasonably high detection probability, we assume that the length of intersection, $x_{\min }$, is equal to the radius of the sensing area of a sensor. Thus, $d \leq r \sqrt{3}$. According to Equation (1), 


$$
\begin{gathered}
d=\frac{2 \pi(r+R)}{N} \\
N=\lambda S=\lambda \pi\left(D^{2}-R^{2}\right)=\lambda \pi\left((R+2 r)^{2}-R^{2}\right)=\lambda \pi\left(4 R r+4 r^{2}\right)=\lambda \pi 4 r(R+r)
\end{gathered}
$$

Putting Equation (4) into Equation (3),

$$
\lambda \geq \frac{1}{2 r^{2} \sqrt{3}} \quad \text { and } \quad \lambda_{\min }=\frac{1}{2 r^{2} \sqrt{3}}
$$

If all the border sensor nodes are activated during $T$, the consumed energy, $E$, is given by

$$
E=N \times T \times E_{\text {active }}
$$

Here $E_{\text {active }}$ is the energy consumed by an active sensor and given by $727.5 \mathrm{~mW}$ [12]. In the proposed scheme, the energy consumed, $E_{p}$, is given by

$$
E_{p}=N \times\left\lfloor\Delta T \times E_{\text {active }}+(N-1) \times \Delta T \times E_{\text {sleep }}\right\rfloor
$$

$E_{\text {sleep }}$ is the energy consumed by a sensor in sleep mode and given by $416.3 \mathrm{~mW}$ [12]. The energy saved by the proposed scheme compared to the DPT algorithm becomes

$$
\Delta E=E-E_{p}=(N-1) \times T \times\left(E_{\text {active }}-E_{\text {sleep }}\right)
$$
thus

Here $N$ is given by Equation (4) and $T$ is given by Equation (2) with $x_{\min }=r$, and

$$
\Delta E=[\lambda \times \pi \times 4 \times r \times(R+r)-1] \times \frac{r}{V_{\max }} \times\left(E_{\text {active }}-E_{\text {sleep }}\right)
$$

From Equations (5)

$$
\Delta E_{\min }=\left[\frac{2 \times \pi \times(R+r)-r \times \sqrt{3}}{V_{\max } \times \sqrt{3}}\right] \times\left(E_{\text {active }}-E_{\text {sleep }}\right)
$$

In Equation (10), the difference between $E$ and $E_{p}$ is the function of target velocity, $V_{\max }$, radius of the sensing area of a sensor, $r$, and radius of tracking area, $R$. In Section 4, the relation between $V_{\max }$ and the detection probability, $p$, will be identified.

We consider only one target above. There might be several targets, however, moving into the sensing area with different velocities and directions. We assume that distribution of the number of targets is Poisson with the rate of $\eta$. The rate the targets are detected in time $T$ is given by

$$
\mu=p \times \eta
$$

If several border sensors detect the presence of a target, several messages are sent to the cluster head simultaneously. The cluster head then has to put the messages into its queue for processing them sequentially. Table 2 summarizes the parameters used in the model for the process of multiple targets. 
Table 2. The parameters used in the process of multiple targets

\begin{tabular}{|c|l|}
\hline Parameter & \multicolumn{1}{c|}{ Description } \\
\hline$\eta$ & The rate of targets moving into the sensing area \\
\hline$\mu$ & The rate of targets detected during $T$ \\
\hline$\gamma$ & The target processing rate \\
\hline$M$ & The average number of messages in the system \\
\hline$t$ & The average target processing time \\
\hline$t_{q}$ & The waiting time in the queue for each message \\
\hline
\end{tabular}

Assume that arrival rate of the messages in the queue is constant and it is equal to the target detection rate, $\mu$. Since only one cluster head processes the target information, this model represents a single server. Using a birth-death system of $\mathrm{M} / \mathrm{M} / 1$ queue, the birth rate is $\mu$ and the death rate is the processing rate $\gamma$. We also assume that the death rate is constant and target population is infinite for the arrival process. To calculate the probability that the queue is in state- $k$ (i.e., has $k$ messages waiting including the one in service), we use the general birth-death formulas.

$$
p_{k}=\left(1-\frac{\mu}{\gamma}\right) \times\left(\frac{\mu}{\gamma}\right)^{k} \quad(\mu<\gamma)
$$

The average number of messages in the system, $M$, is

$$
M=\frac{\delta}{1-\delta},\left(\delta=\frac{\mu}{\gamma}\right), M=\mu \times t, t=\frac{\frac{1}{\gamma}}{1-\delta}, t_{q}=\frac{\frac{\mu}{\gamma^{2}}}{1-\delta}
$$

\section{Performance Evaluation}

In this section we present the simulation results evaluating the performance of the proposed scheme. The simulation study mainly focuses on the energy saved by the proposed scheme compared to the DPT algorithm, the detection probability, and the message processing time (delay time) as the number of targets varies.

We simulated a scenario where a target moves with random velocity. The simulator was developed to detect the target, considering the detection probability and energy consumed. The simulation program is a discrete-event simulator developed using $\mathrm{C}$ language. In the simulation we distribute the sensors uniformly over a cluster with $D$ $=120 \mathrm{~m}$ and $r=10 \mathrm{~m}$. The velocity of target movement varies randomly from $0 \mathrm{~m} / \mathrm{s}$ to $60 \mathrm{~m} / \mathrm{s}$ in order to evaluate its impact on the performance of the proposed scheme.

Figure 3 shows the energy saved by the proposed scheme compared to the DPT scheme obtained using Equation (10). The amount of energy saved decreases as the velocity of target increases. This is because the border sensor nodes need to fastly on and off if the target moves fastly, and thus energy consumption increases compared to the case of slow target. Note, however, that the velocity here is up to 100 meter per second. For typical targets of below the 20 or $30 \mathrm{~m} / \mathrm{s}$ speed, the energy saving is very significant. 


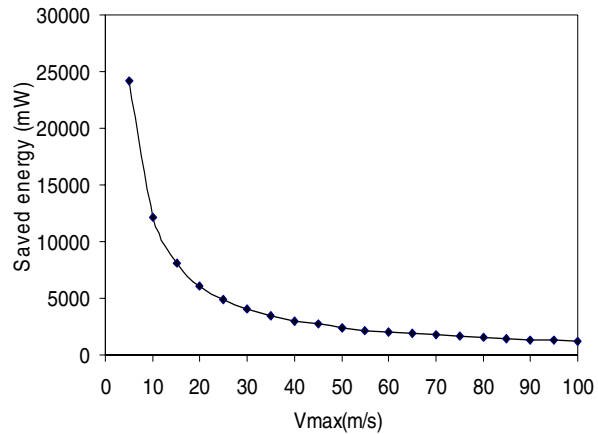

Fig. 3. The amount of energy saved by the proposed scheme as the target speed varies

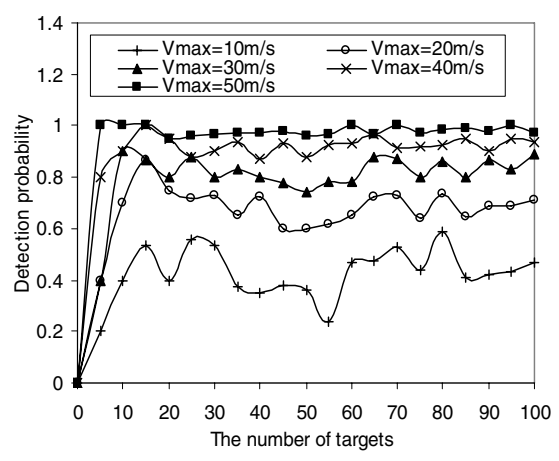

Fig. 4. Detection probability vs. number of targets

Figure 4 presents the relationship between the detection probability and the number of targets. As the threshold velocity of target increases from $10 \mathrm{~m} / \mathrm{s}$ to $50 \mathrm{~m} / \mathrm{s}$, the detection probability increases. When the velocity of the target is smaller than the threshold velocity, the number of missed targets decreases since the rotation time $T$ is small and the period that the border sensors are activated is short. However, the amount of energy saved becomes small for high threshold velocity. Hence, we need to choose a proper period so that the detection probability is high while energy saving at the border sensor nodes is substantial.

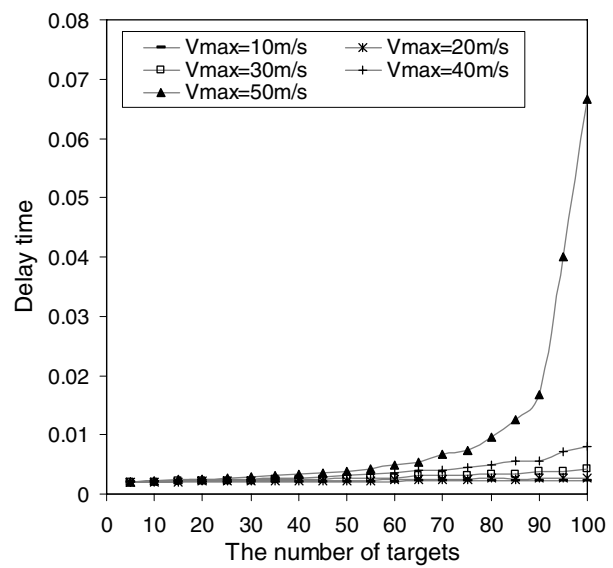

Fig. 5. The relationship between the delay time and the number of targets

In Figure 5, the relationship between the delay (processing time) and velocity of the target is shown. As the velocity changes from $10 \mathrm{~m} / \mathrm{s}$ to $40 \mathrm{~m} / \mathrm{s}$, the delay increases slowly. For $V=50 \mathrm{~m} / \mathrm{s}$, the delay increases rapidly when the number of targets becomes larger than 90 . The reason for this is the ratio between the death rate and birth rate, $\delta=\mu / \gamma$, becomes close to 1 . 


\section{Conclusion and Future Work}

We have proposed a new scheme for target detection with a sensor network. The border sensors detect the targets moving into the sensing area and inform the cluster head in their cluster. The proposed scheme allows significant energy saving by letting the border sensors awake shortly one after another in a circular fashion. As a result, the lifetime of the sensors can be prolonged. Additionally, in case of multiple-target tracking, we have found the relationship between the processing time at the cluster head and the number of targets.

In this paper we have assumed that there exists only one cluster head. In the future we will consider the case of multiple clusters in energy efficient target detection. We will also investigate the impact of other parameters on the target detectability.

\section{References}

[1] J. Nemeroff, L. Garcia, D. Hampel, and S. DiPierro. Application of sensor network communications. In military Communications Conference, 2001.

[2] S. Bandyopadhyay and E.J. Coyle. An Energy Efficient Hierarchical Clustering Algorithm for Wireless Sensor Networks. INFOCOM 2003. Twenty-Second Annual Joint Conference of the IEEE Computer and Communications Societies. IEEE.

[3] Y. Xu and W. C. Lee. On localized prediction for power efficient object tracking in sensor networks. In Proceeding 1. st. International Workshop on. Mobile Distributed Computing (MDC), Providence, Rhode Island, May 2003.

[4] H. Yang and B. Sikdar. A protocol for tracking mobile targets using sensor networks. Proceedings of IEEE Workshop on Sensor Network Protocols and Applications, 2003.

[5] B. Krishnamachari, S. B. Wicker and R. Berjar. Phase transition phenomena in wireless ad-hoc network, proceeding of IEEE GLOBECOM, San Atonio, TX, November 2001.

[6] S. Banerjee and S. Khuller. A clustering scheme for hierarchy control in multi-hop wireless networks. Proceedings of IEEE INFOCOM, April 2001.

[7] F. Zhao, J. Shin and J. Reich. Information-driven dynamic sensor coloration for target tracking, IEEE signal processing maganize, vol. 19, no. 2, pp 61-77. March 2002.

[8] D. Li, K. Wong, Y.H. Hu and A. Sayeed. Detection, classification and tracking of targets in distributed sensor networks. IEEE Signal Processing Maganize, vol 9, no.2, March 2002.

[9] L.M. Kaplan, Q. Le, and P. Molnar. Maximum likehood methods for bearing-only target localization. Proceedings of IEEE ICASSP, pp. 554-557, May, 2001.

[10] J.S. Scholl, L.P. Clare, and J.R. Agre. Wavelet packet-based target classification schemes. Meeting of IRIS Specialty Group on Acoustic and Seismic Sensing, laurel, MD, September, 1998.

[11] K. Yao, et. al., Estimation and tracking of an acoustic/seismic source using a beamforming array based on residual minimizing methods. Proceedings of IRIA-IRIS, pp. 153-163, January 2001.

[12] V. Raghnathan, et. al., Energy aware wireless microsensor networks. IEEE Signal Processing Magazine, March 2002. 